


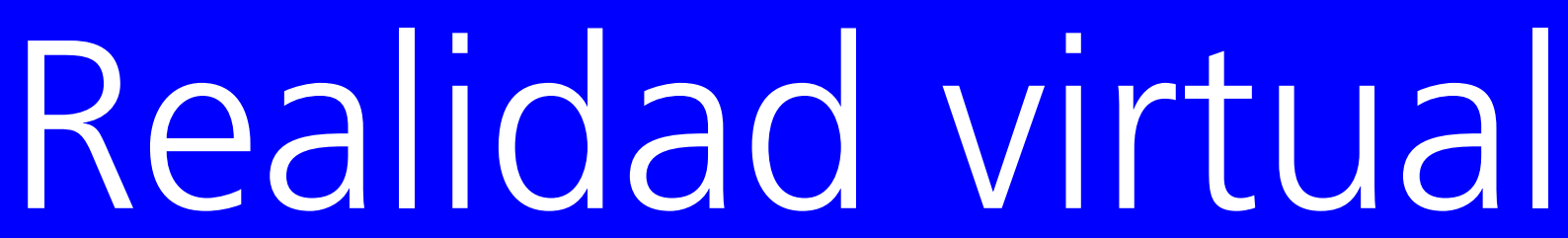

y realidad

aumentada

en la educación,

una instantánea

nacional

e internacional

Alejandro Gustavo Piscitelli Altomari 


\section{Realidad virtual y realidad aumentada en la educación, una instantánea nacional e internacional}

\section{Alejandro Gustavo Piscitelli Altomari}

Filósofo por la Universidad Nacional de Buenos Aires, titular del Laboratorio de Innovación Pedagógica en la Carrera de Ciencias de la Comunicación en la Universidad Nacional de Buenos Aires. apiscite@well.com

Fecha de recepción: 23 de noviembre, 2016

Fecha de aceptación: 07 de marzo, 2017

\section{Resumen}

En este artículo se presenta el estado del arte de una investigación de largo alcance cuyo propósito consiste en evaluar una serie de proyectos de realidad aumentada y realidad virtual en términos de efemeralización educativa. ¿Qué proyectos se están desarrollando alrededor del mundo? ¿Qué países cuentan con iniciativas de esta naturaleza? ¿Qué valores agregados destacan estos proyectos? ¿Se identifican o no indicios de efemeralización educativa en la revisión preliminar de los proyectos? ¿Qué siguientes pasos se recomiendan para su evaluación?, son las interrogantes que aquí se abordan. Se concluye que el atributo de valor más reiterado es la divulgación amplia de contenidos que de otra manera serían inaccesibles para los usuarios, que en sí mismo no garantiza el óptimo aprovechamiento de la experiencia ni la efemeralización del proceso educativo, salvo que los contenidos se integren a un ecosistema congruente con dicha visión.

Palabras clave | educación; tecnología; realidad virtual; realidad aumentada; efemeralización 
REALIDAD VIRTUAL Y REALIDAD AUMENTADA

EN LA EDUCACIÓN, UNA INSTANTÁNEA

NACIONAL E INTERNACIONAL

\section{Abstract}

This paper presents the State of the art of a long range research which purpose is to assess a serie of projects of augmented reality and virtual reality, in terms of educational ephemeralization. Which projects that are being developed around the world? Which countries have this kind of initiatives? What added values highlight these projects? There's evidence of educational ephemeralization in the preliminary review of these projects, or not? Which following steps are recommended for their evaluation? These are the questions addressed here. It is concluded that the most repeated value attribute is the dissemination of content that otherwise would be inaccessible to the users, which, in itself, does not guarantee the optimal use of experience or the ephemeralization of the educational process, unless contents are integrated into a consistent ecosystem with this vision.

Keywords | education; technology; virtual reality; augmented reality; ephemeralization 
REALIDAD VIRTUAL Y REALIDAD AUMENTADA

EN LA EDUCACIÓN, UNA INSTANTÁNEA

NACIONAL E INTERNACIONAL

\section{Introducción}

Como parte de una investigación mayor, en este documento se exponen los resultados de una exploración realizada durante el 2016 con el propósito de sintetizar un estado de la cuestión acerca de los usos educativos que la realidad aumentada y la realidad virtual describen actualmente en México y el resto del mundo. Las preguntas de investigación que se abordaron son las siguientes: ¿Qué proyectos se están desarrollando alrededor del mundo? ¿Qué países cuentan con iniciativas de esta naturaleza? ¿Qué valores agregados destacan estos proyectos? ¿Se identifican o no indicios de efemeralización educativa en la revisión preliminar de los proyectos? ¿Qué siguientes pasos se recomiendan para su evaluación?

Para abordar estas interrogantes, se entenderá por efemeralización el incremento en la eficiencia de un proceso mediante la reducción de materiales utilizados y el uso de estos en sus formas más eficaces, siguiendo a Richard Buckminster Fuller (Baldwin, 1997, 15). Es decir, hacer más con menos en los procesos de enseñanza-aprendizaje. En lo que refiere a la realidad aumentada, entenderemos aquella solución tecnológica en la que se "(...) brinda al usuario información adicional de otra manera imperceptible para los sentidos humanos, por medio de estímulos artificiales superpuestos sobre objetos del mundo real (...)" (Sherman \& Craig, 2003, 43) 1 .

Finalmente, la realidad virtual (RV) se entenderá como:

(...) un medio interactivo compuesto por simulaciones de computadora, que detecta la posición y las acciones del participante y reemplaza o aumenta la respuesta a uno o más sentidos, dando la sensación de estar mentalmente inmerso o en la simulación un mundo virtual (...) (Sherman \& Craig, 2003, 38)2. 
REALIDAD VIRTUAL Y REALIDAD AUMENTADA

EN LA EDUCACIÓN, UNA INSTANTÁNEA

NACIONAL E INTERNACIONAL

A los conceptos clave antes expuestos añadiremos el de inmersión, experiencia sensorial "(...) lograda mediante la presentación de un mundo virtual a los usuarios, que, basado en su ubicación y su orientación, proporciona estímulos artificiales a uno o más de sus sentidos en respuesta a su posición y sus acciones (...)" (Sherman \& Craig, 2003, 382). También se retomará la noción de ambiente virtual, que de acuerdo con Jeffrey Jacobson (2008), "(...) es un espacio artificial, un mundo imaginario o ilusorio, creado y mantenido por aplicaciones informáticas adecuadas, con el que el usuario interactúa y en el que también puede interactuar con objetos, agentes o representaciones de otros usuarios encontrados en el mismo" (4), en el que el usuario experimenta "la sensación de estar allí en el mundo virtual y de que este es su entorno, en lugar del mundo real, o quizás además de él" (6).

You-tian Ye (2016) escala la realidad virtual a terrenos de interdisciplinariedad, considerando la interacción del usuario por medio de sus sentidos al mencionar que los entornos de realidad virtual ofrecen un nuevo tipo de interacción multimodal en la cual las personas pueden sentir de diversas maneras la simulación virtual hecha por computadora y que "el desarrollo de la realidad virtual es una técnica interdisciplinaria basada en gráficos y simulación creados por computadora, la interacción hombre-máquina, tecnología multimedia y de los sentidos" (49). Aseveración que puede aplicarse también a los entornos de realidad aumentada.

\section{Resultados}

Para conformar el estado del arte, durante el 2016 se realizó una pesquisa en repositorios especializados como Gale, EBSCO, JSTOR, Proquest, así como búsquedas simples en Google y consultas semiestructuradas a especialistas en el tema; los parámetros de búsqueda que guiaron la conformación del universo no exhaustivo de casos de interés, fueron:

1 Proyectos nacionales e internacionales que involucren tecnologías de realidad virtual, realidad aumentada o inmersión, con fines educativos, aun cuando la educación no haya sido su propósito original. 
2 Que hayan tenido origen lo mismo en contextos académicos que empresariales.

3 Que en algún sentido los proyectos contribuyan a la efemeralización de los procesos de enseñanza-aprendizaje.

4 Que cuenten con una longevidad de mínimo un año y se encuentren activos y/o en desarrollo para su perfeccionamiento luego de haber sido aplicados, al cierre de esta etapa de la investigación.

Con este marco en mente, se realizó una búsqueda que arrojó cuarenta y cinco iniciativas de 1998 a la fecha, de las que finalmente se eligieron treinta de especial interés por su estrecha relación con o aplicación exclusiva en el ámbito educativo, mismos que constituyen el universo que a continuación se presenta.

\section{1 | Alchemy VR}

Tipo | privado

Descripción | empresa dedicada a la creación de contenidos de realidad virtual para el aprendizaje.

Contribución | eficienta la divulgación de conocimientos científicos, culturales y tecnológicos complejos, por medio de experiencias que ofrecen al usuario contenidos a los que de otra forma no podría acceder, de forma lúdica y atractiva; por ejemplo, viajar en el espacio acompañando al astronauta británico Tim Peake, en un descenso en realidad virtual, desde la estación espacial internacional a las estepas de Kazajistán, en la nave Soyuz TMA - 19M, a través del Space Descent VR with Tim Peake, en el Museo de Ciencias de Londres.

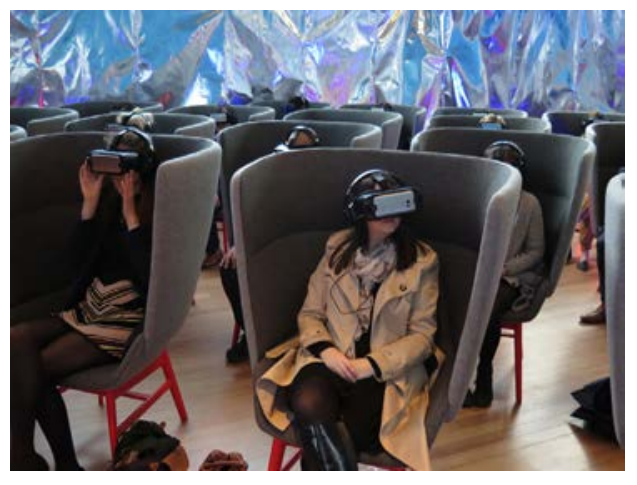

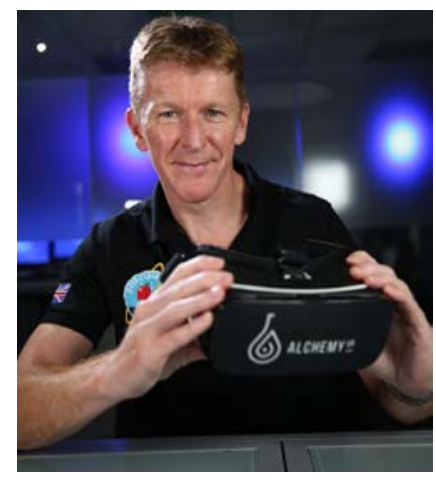

Imagen 1. Visitantes en el Museo de la Ciencia de Londres.

Imagen 2. El astronauta británico Tim Peake, presentando el proyecto realizado con Alchemy VR para el Museo de Ciencias de Londres. 
Tecnología | realidad virtual, inmersión

Status | proyectos activos y nuevos en desarrollo

Web | alchemyvr.com/

País | Reino Unido

Año | 2014

Auspiciante | Atlantic Productions

\section{2 | ARToolKit}

Tipo | académico

Descripción | desarrollado originalmente por Hirokazu Kato en 1992 y publicado por el HIT Lab de la Universidad de Washington. Se trata de una biblioteca que permite la creación de aplicaciones de realidad aumentada, en las que se sobrepone imágenes virtuales al mundo real. Actualmente es una herramienta que se mantiene como proyecto de código abierto, alojado en SourceForge con licencias comerciales disponibles en ARToolWorks

Contribución | facilita el proceso educativo al permitir la difusión de contenidos a mayor escala, con menos herramientas e involucrando la creatividad del usuario.

Tecnología | realidad aumentada

Status | proyecto activo

Web | artoolkit.org/

País | Estados Unidos

Año | 1999

Auspiciante | Human Interface Technology Lab (HITLab), University of Washington y ARToolworks, Inc.

\section{3 | Augment}

Tipo | privado

Descripción | plataforma de realidad aumentada que permite a los estudiantes y profesores visualizar modelos en tres dimensiones (3D) en el entorno real, en tiempo real y en gran escala. Originalmente creada con fines comerciales, Augment ha encontrado una importante aplicación en el campo educativo como son: su uso en la enseñanza de ingeniería 
REALIDAD VIRTUAL Y REALIDAD AUMENTADA

EN LA EDUCACIÓN, UNA INSTANTÁNEA

NACIONAL E INTERNACIONAL

mecánica en la Universidad de Harran, en Sanliurfa, Turquía; el Royal Melbourne Institute of Technology (RMIT) Campus Vietnam, que ha empleado Augment en su programa de medios digitales para complementar la enseñanza de la historia.

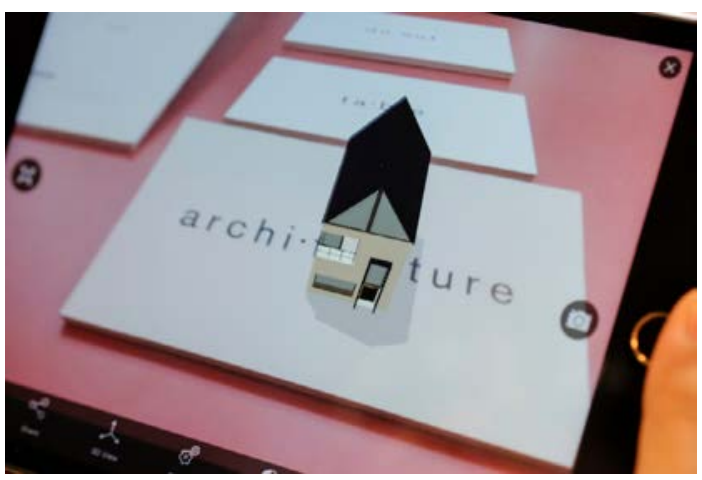

Contribución | vuelve accesible la información en alta calidad a una mayor cantidad de usuarios, facilitando así las presentaciones, conferencias, exposiciones y el trabajo en aula. Ofrece suscripciones gratuitas para los estudiantes, los maestros y las instituciones académicas, por lo que con menos recursos se pueden obtener resultados de mucho mejor calidad e innovadores.

Tecnología | realidad aumentada, 3D

Status | proyectos activos y nuevos en desarrollo

Web | augment.com

País | Francia

Año | 2011

Auspiciante | Augment

\section{4 | Aula Virtual Móvil}

Tipo | híbrido

Descripción | aula móvil de realidad virtual, primera en su tipo en México, que utiliza tecnología de última generación para lograr experiencias sensoriales que inducen al aprendizaje de la ciencia y la cultura, de una manera diferente y divertida. Ofrece contenidos didácticos que emulan viajes por el sistema solar y Marte, o un recorrido virtual en Fuerte de Bacalar, por ejemplo. 

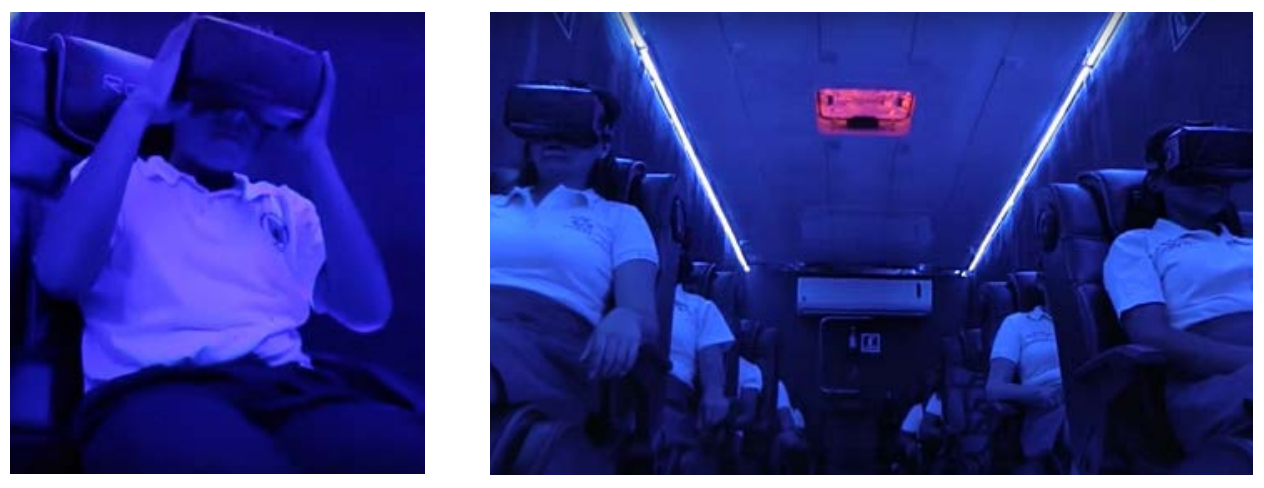

Imagen 4. Aula virtual móvil Hypnogic CONQUICYT.

Contribución | ofrece la posibilidad de realizar recorridos virtuales a lugares normalmente inaccesibles o simplemente para poder vivirlos de manera más profunda y completa. Simplifica el proceso de enseñanza aprendizaje, acercando contenidos académicos a los usuarios, a fin de despertar el interés y lograr la apropiación del conocimiento mediante la experiencia sensorial, especialmente entre niños y jóvenes de comunidades que aún tienen un acceso limitado o inexistente a espacios de divulgación científico-tecnológica.

Tecnología: realidad virtual

Status | prototipo activo, proyecto en desarrollo

Web | youtube.com/watch?v=Lu2fIIZdQ_Q

País | México

Año| 2016

Auspiciante | Hypnogic para el Consejo Quintanarroense de Ciencia y Tecnología (COQCYT)

\section{5 | CAVE2}

Tipo | académico

Descripción | CAVE2, son instalaciones de próxima generación híbrida dos y tres dimensiones (2D y $3 D$ ); entorno de realidad virtual que combina la experiencia de la plataforma de inmersión Monash en computación de alto rendimiento, con gráficos de computadora y redes, para representar conjuntos de terascale de claridad sin precedentes. Como visor de microscopio del siglo XXI, CAVE2 permite la exploración interactiva de datos de fuentes como el Sincrotrón australiano (acelerador de partículas), microscopios, instrumentos médicos de proyección de imagen, etcétera. 
REALIDAD VIRTUAL Y REALIDAD AUMENTADA

EN LA EDUCACIÓN, UNA INSTANTÁNEA

NACIONAL E INTERNACIONAL

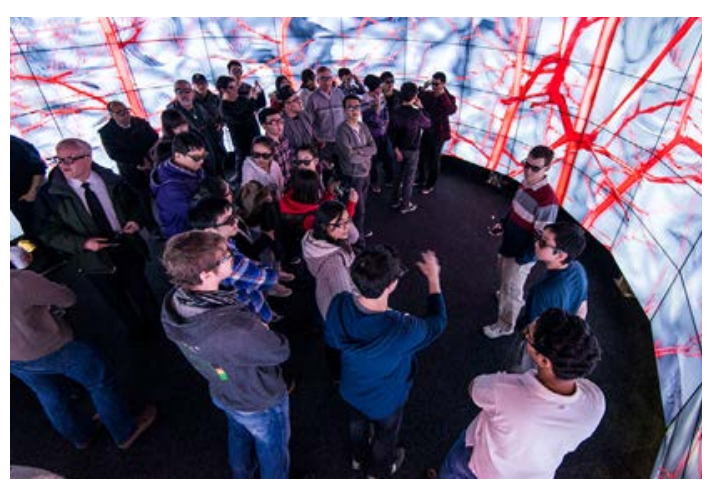

Contribución | proporciona acceso a un vasto número de contenidos a través de una experiencia inmersiva completa. De lo más desarrollado en materia de escenarios virtuales para inmersión.

Tecnología | inmersión, realidad virtual

Status | activo

Web | monash.edu/mivp/index.php?option=com_content\&view=article\&id=15\&ltemid=101

País | Estados Unidos

Año $\mid 2013$

Auspiciante | Monash University's Monash Technology Research Platforms.

\section{6 | 3D Cyber Anatomy Room}

Tipo | híbrido

Descripción | entorno de inmersión para experiencia 3D de disección de la anatomía humana. La anatomía fue desarrollada en conjunto con el Netter Anatomy Group y el software con Elsevier y es un proyecto muy completo que incluye: esqueleto, articulaciones, ligamentos, sistema muscular, sistema nervioso y cerebro, entre otros.

(Video del 3D Cyber Anatomy Room)

Contribución | permite mejorar el proceso educativo al lograr una mayor y más eficaz comprensión de temas complejos, pues ofrece al usuario una experiencia integral y sensible, en menos tiempo y en un solo espacio. Permite que el conocimiento sea una experiencia integral y sensible.

Tecnología | realidad virtual, inmersión 
REALIDAD VIRTUAL Y REALIDAD AUMENTADA

EN LA EDUCACIÓN, UNA INSTANTÁNEA

NACIONAL E INTERNACIONAL

Status | proyecto activo

Web | cyberscience3d.com/project/cyber-anatomy-room/

País | Estados Unidos

Año | 2015

Auspiciante | Cyber-Anatomy Inc.

\section{7 | EON Reality}

Tipo | híbrido

Descripción | centro digital interactivo creado para producir contenido virtual y de realidad aumentada, y capacitar a futuros profesionales en ambos recursos. Sus proyectos abarcan distintas áreas del conocimiento, por ejemplo: el viaje de Howard Carter en el Valle de los Reyes, Egipto; principios de armado y mantenimiento del motor diesel mediante una serie de lecciones de realidad virtual gamificada, entrenamiento médico para la práctica oftalmológica en el aula, simulador de la anatomía humana, etcétera.

(Video de inmersión virtual con tecnología de EON Reality)

Contribución | facilita la divulgación educativa por medio de soluciones de realidad virtual que involucran activamente a los alumnos, dando como resultado un aprendizaje más rápido, una mejor retención y la mejor toma de decisiones con base en el conocimiento adquirido. Ofrece soluciones de costo accesible, que permiten a los estudiantes de secundaria aprender de la experiencia práctica y sin simuladores caros ni disecciones de mamíferos y anfibios.

Tecnología | realidad virtual, inmersión, realidad aumentada

Status | activo, nuevos proyectos en desarrollo

Web | eonreality.com/

País | Estados Unidos

Año | 2016

Auspiciante | EON 


\section{8 | Erechtheum}

Tipo | académico

Descripción | exploración en realidad virtual del Erecteión en Atenas, se aplicó como piloto a grupos de $4^{\circ}$ grado de una escuela primaria de Atenas; constituye una enseñanza a través del uso de diferentes representaciones creadas con el lenguaje VRML (lenguaje para modelar mundos virtuales en 3D). La aplicación también tiene como objetivo hacer hincapié en las ventajas significativas de la realidad virtual como una forma eficaz de ofrecer materiales de estudio.

Contribución | permite a los alumnos realizar un recorrido y conocer un sitio histórico y acceder a mayor información y de mayor profundidad, en menor tiempo. Genera interés en el estudio de la historia y lo convierte en un proceso divertido e integral. Los resultados del estudio mostraron un incremento en el rendimiento cognitivo de los estudiantes y mejor asimilación de cuestiones relativas a la arquitectura y la geometría del espacio.

Tecnología | realidad virtual, lenguaje de modelado de mundos virtuales en tres dimensiones (VRML)

Status | inactivo, en desarrollo

Web | ijedict. dec.uwi.edu/viewarticle. php?id=325\&layout=html

País | Grecia

Año | 2007

Auspiciante | Research Academic Computer Technology Institute \& University of the Aegean

\section{9 | Estudiofuture}

Tipo | privado

Descripción | empresa dedicada al desarrollo de experiencias inmersivas, 3D, de realidad virtual y realidad aumentada. Ha creado distintos programas educativos para el entrenamiento seguro en áreas de ingeniería, recorridos virtuales por lugares de la antigüedad y otros diseñados para aprovechar la realidad virtual en la enseñanza de la historia. 
REALIDAD VIRTUAL Y REALIDAD AUMENTADA

EN LA EDUCACIÓN, UNA INSTANTÁNEA

NACIONAL E INTERNACIONAL

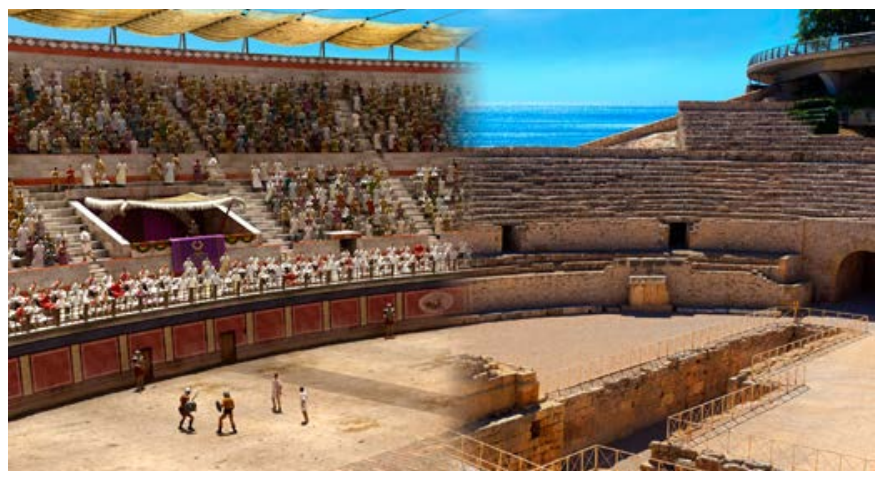

Imagen 7. Tarraco VR una experiencia de Estudiofuture

Contribución | disemina contenidos académicos de acceso abierto. Promueve el uso de nuevas tecnologías en el proceso de enseñanza.

Tecnología | realidad virtual

Status | activo y en desarrollo de nuevos proyectos

Web | estudiofuture.com

País | España

Año | 2016

Auspiciante | Estudiofuture

\section{0 | Google Expeditions}

Tipo | privado

Descripción | plataforma para el uso de la realidad virtual en las aulas. Permite realizar excursiones escolares virtuales y tomar clase en distintos lugares del planeta.

Contribución | promueve la difusión de conocimientos a mayor escala, permite a los alumnos el acceso a lugares distantes y motiva a los maestros a guiar una clase teniendo como aula virtual y escenario, alguno de los lugares a los que de otra forma sería complicado llevar a su grupo: en un momento pueden estar en la Antártida, en otro tomar clase recorriendo la zona de Machu Pichu, en Perú, y después estar en el espacio.

Tecnología | realidad virtual

Status | activo

Web | edu.google.co.in/expeditions

País | Estados Unidos 
REALIDAD VIRTUAL Y REALIDAD AUMENTADA

EN LA EDUCACIÓN, UNA INSTANTÁNEA

NACIONAL E INTERNACIONAL

Año | 2016

Auspiciante | Google

\section{1 | HIT Lab. Learning in Virtual Environments}

Tipo | académico

Descripción | proyecto de investigación cuyo objetivo general es determinar si los entornos virtuales inmersivos mejoran la comprensión de los procesos ambientales complejos en comparación con lo que se puede aprender de las simulaciones en computadora, no inmersivas. Contribución | será concluir qué tanto y cómo es que la introducción de la realidad virtual como una de las principales herramientas didácticas, contribuye a la mejora de la educación. Tecnología | realidad virtual, inmersión

Status | activo

Web | hitl.washington.edu/projects/learnve/

País | Estados Unidos

Año| 2006

Auspiciante | Human Interface Technology Lab (HITLab), University of Washington

\section{2 | Ixtli}

Tipo | académico

Descricpión | Ixtli es el Observatorio de Visualización de Realidad Virtual de la UNAM, infraestructura de realidad virtual inmersiva que permite a los usuarios trabajar con ambientes tridimensionales interactivos y dispositivos de interacción natural, para el apoyo de la labor en áreas de docencia, investigación y difusión.

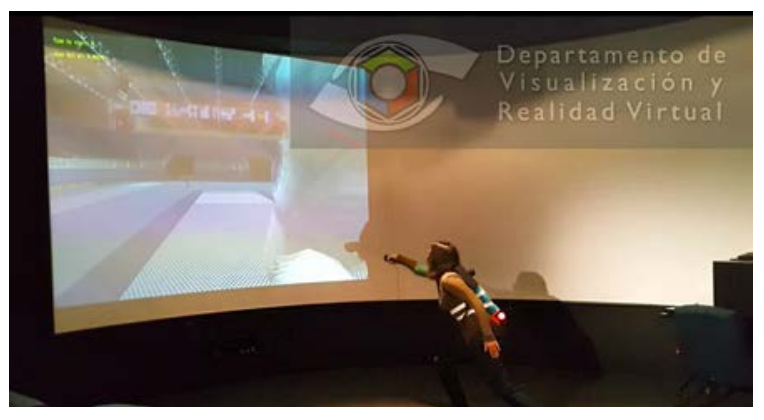


Contribución | asiste a la comunidad académica en los procesos de uso y desarrollo de sistemas interactivos 3D y de realidad virtual, en apoyo a la labor académica y de investigación. Acerca una mayor cantidad de contenidos al usuario y ofrece una experiencia única, gracias a la realidad virtual.

Tecnología | realidad virtual, inmersión.

Status | activo, nuevos proyectos en desarrollo

Web | ixtli.unam.mx/

País | México

Año | 2004

Auspiciante | Departamento de Visualización y Realidad Virtual, Universidad Nacional Autónoma de México (UNAM)

\section{3 | Laboratorio Nacional de Visualización Científica Avanzada (LNVCA)}

Tipo | híbrido

Descripción | espacio equipado con una unidad de visualización de alta resolución en dos dimensiones (2D), otra OMNIGlobe (sistema esférico de proyección para la visualización de datos globales) y una unidad de visualización inmersiva 3D tipo Cave. Único en su tipo en América Latina, tiene como objetivo brindar herramientas de supercómputo y visualización científica avanzada que sirvan de apoyo a las actividades de docencia e investigación.
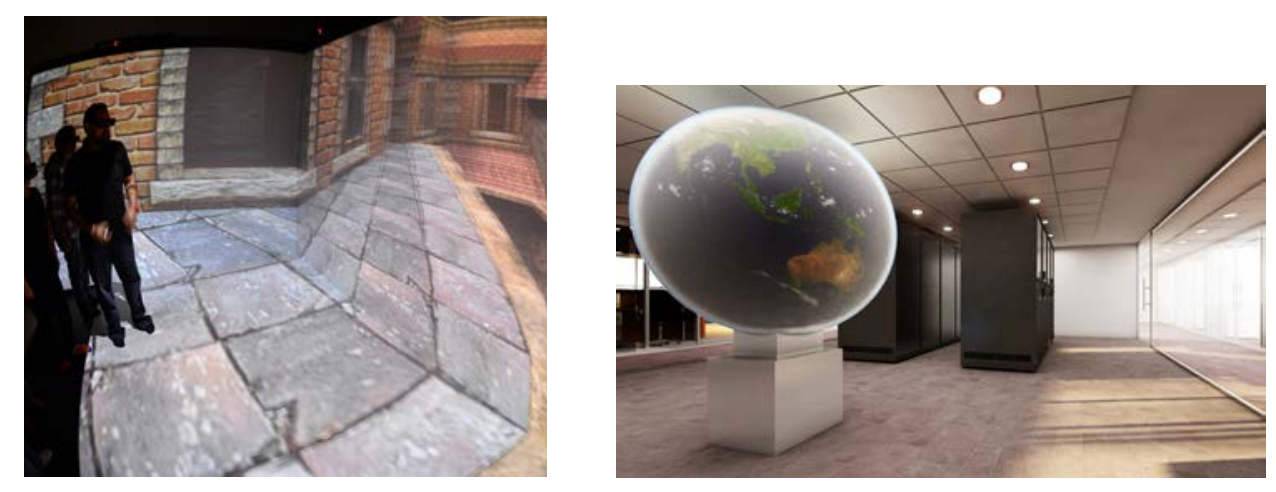

Imagen 9. Inmersión Virtual en el Laboratorio de visualización Científica Avanzada de la UNAM.

Imagen 10. Visualización esférica en el Laboratorio Nacional de Visualización Científica Avanzada (LNVCA) de la UNAM.

Contribución | apoya la diseminación de conocimientos a estudiantes de todos los niveles educativos, favorece una mejor y más rápida transmisión de información compleja, hacién- 
REALIDAD VIRTUAL Y REALIDAD AUMENTADA

EN LA EDUCACIÓN, UNA INSTANTÁNEA

NACIONAL E INTERNACIONAL

dola accesible al usuario. Permite el almacenamiento de bases de datos de gran volumen, de alta disponibilidad y alta seguridad.

Tecnología | visualización en dos y tres dimensiones, visualización esférica, realidad virtual e inmersión.

Status | activo

Web | geociencias.unam.mx/ucav/about/lnvca/lnvca.php

País | México

Año | 2016

Auspiciante | Universidad Nacional Autónoma de México (UNAM) y Universidad Autónoma de Querétaro (UAQ).

\section{4 | NICE}

Tipo | académico

Descripción | proyecto educativo que consiste en un entorno de aprendizaje inmersivo para niños relacionado con tecnologías de realidad virtual multiusuario. Ofrece un entorno atractivo donde los niños construyen ecosistemas virtuales simples de cultivar, colaboran a través de redes con otros niños ubicados de manera remota y crean historias a partir de sus interacciones en el mundo real y virtual.
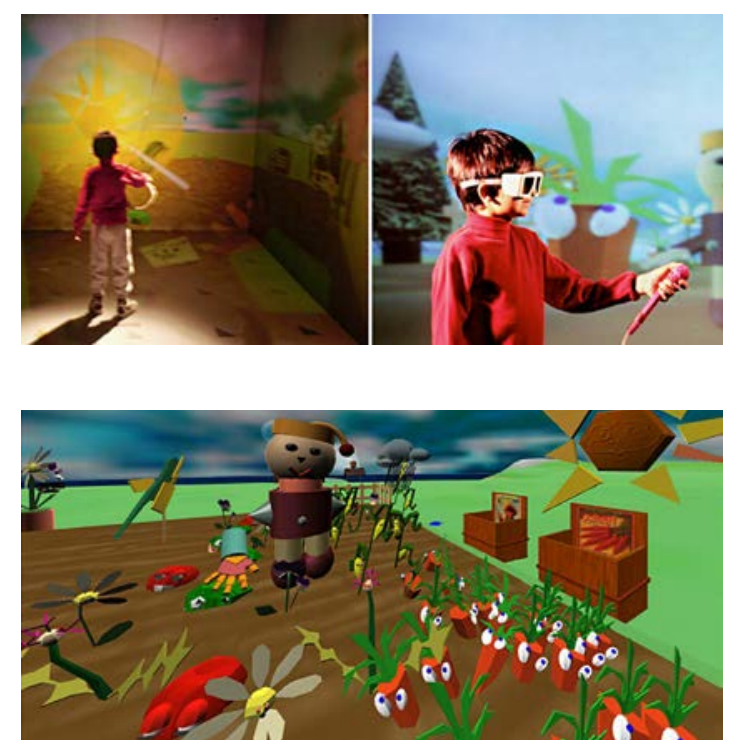

Imagen 11. NICE, ecosistema virtual para niños.

Imagen 12. Un Avatar planta zanahorias en el jardín de NICE. M. Roussou. 
REALIDAD VIRTUAL Y REALIDAD AUMENTADA

EN LA EDUCACIÓN, UNA INSTANTÁNEA

NACIONAL E INTERNACIONAL

Contribución | permite al alumno recibir la información de forma más interactiva, dinámica y visual.

Tecnología | realidad virtual, inmersión

Status | actualmente inactivo, en desarrollo

Web | evl.uic.edu/documents/nices97.pdf

País | Estados Unidos

Año| 1998

Auspiciante | Electronic Visualization Laboratory \& Interactive Computing Environments Laboratory, University of Illinois

\section{5 | PublicRV}

Tipo | híbrido

Descripción | organización "virtual" sin fines de lucro, dedicada al desarrollo y la investigación en software relacionado con la realidad virtual en la educación. Crea programas de realidad virtual por contrato o en coordinación con instituciones académicas y filiales alrededor del mundo. Los temas que maneja son científicos, culturales e históricos, principalmente de Grecia, Roma y Egipto.

Contribución | promueve la diseminación del conocimiento a una mayor escala, permite el acceso a la información compleja de una forma atractiva, para estudiantes de nivel primaria y secundaria principalmente.

Tecnología | realidad virtual

Status | activo

Web | publicvr.org/html/about.html

País | Estados Unidos

Año | 2012

Auspiciante | Public RV

\section{6 | Reality Deck. Immersive Gigapixel Display}

Tipo | académico

Descripción | instalaciones de visualización inmersiva para exploración y manipulación de 
datos, situadas en el Centro de excelencia en tecnología inalámbrica y de la información, de la Universidad de Stony Brook; es una pantalla inmersiva de 1,5 gigapixel en mosaico con un campo horizontal completo de 360 grados de vista, conformada por 416 pantallas LCD de alta densidad, construida para abordar problemas de big data de actualidad.

Contribución | rompe las barreras en la visualización de datos y ayuda a los científicos a hacer frente a los retos presentados por los grandes conjuntos de datos de hoy y mañana.

Tecnología | realidad virtual, visualización inmersiva, realidad aumentada

Status | activo

Web | labs.cs.sunysb.edu/labs/vislab/reality-deck-home/

País | Estados Unidos

Año | 2011

Auspiciante | Center of Excellence in Wireless and Information Technology, Stony Brook University con el apoyo de National Science Foundation.

\section{7 | REVIR (Realidad Virtual)}

\section{Tipo | académico}

Descripción | consiste en ofrecer al alumnado y al profesorado de secundaria de Cataluña sesiones de trabajo experimental en un laboratorio informatizado en el Centro de Investigación para la Educación Científica y Matemática (CRECIM) de la Universidad Autónoma de Barcelona (UAB). Las sesiones están enfocadas a las materias de física, química y biología, y a contenidos de relevancia social, como son: la salud, la educación para la seguridad vial y la energía.

Contribución | hace posible la observación directa del trabajo que realizan los estudiantes durante la práctica y el análisis posterior de la información recopilada en dossiers y formularios. Asimismo, este proyecto ayuda a los estudiantes a construir conocimientos, transferirlos a nuevos contextos y reforzar su comprensión de los temas científicos, por medio de un acercamiento sensible a través de las tecnologías de la información y la comunicación (TIC), que fomenta en los jóvenes la inquietud de investigar y el pensamiento crítico.

Tecnología | realidad virtual, sensores digitales, sistemas de captación de datos en tiempo real, simulaciones. 
REALIDAD VIRTUAL Y REALIDAD AUMENTADA

EN LA EDUCACIÓN, UNA INSTANTÁNEA

NACIONAL E INTERNACIONAL

Status | activo, en desarrollo

Web | crecim.uab.cat/revir/

País | España

Año | 2016

Auspiciante | Universidad Autónoma de Barcelona

\section{8 | Second Life}

Tipo | híbrido

Descripción | plataforma educativa de entornos virtuales para el aprendizaje, inmersivos en tres dimensiones, que reúne a más de 700 instituciones educativas que han creado programas de aprendizaje conjunto.

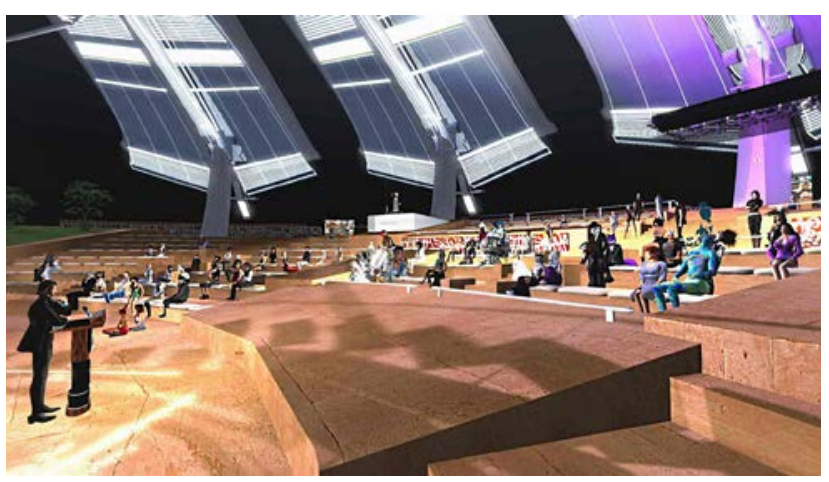

Contribución | permite a los estudiantes trabajar tanto de manera individual como en equipo y tomar clase en entornos virtuales que hacen posible la colaboración entre personas e instituciones educativas de todo el mundo.

Tecnología | realidad virtual, inmersión 3D

Status | activo

Web | lecs-static-secondlife-com.s3.amazonaws.com/work/SL-Edu-Brochure-010411.pdf

País | Estados Unidos

Año | 2003

Auspiciante | Linden Lab 


\section{9 | Sistema de Museos Virtuales}

Tipo | académico

Descripción | el Sistema de Muesos Virtuales de la Universidad Autónoma Metropolitana, publica y hace comunicable el conocimiento, procura la expansión de la cultura universal a través del ciberespacio. Es un espacio cibernético de educación pública alternativa para la difusión del diseño, las ciencias y las artes.

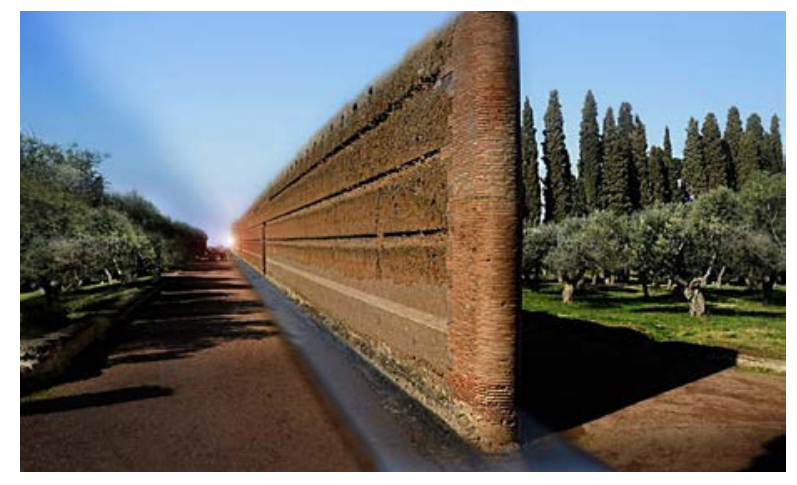

Imagen 14. Museos virtuales UAM, México.

Contribución | fomenta en el usuario el placer de realizar exploraciones propias, análogas o divergentes, a través de proyectos integradores y de fusión, para ser a su vez, integrados en redes con intereses afines: educación, diseño, arte, ciencia. Colabora en la innovación de la oferta de educativa, promueve el intercambio de información y provee elementos para la generación y difusión de conocimientos, mediante el aprovechamiento de las facilidades ofrecidas por las tecnologías de visualización, información y comunicación (TVIC). Ayuda a la diseminación del conocimiento.

Tecnología | realidad virtual, inmersión

Status | activo

Web | museosvirtuales.azc.uam.mx/smv/

País | México

Año | 2004

Auspiciante | Universidad Autónoma Metropolitana (UAM) 
REALIDAD VIRTUAL Y REALIDAD AUMENTADA

EN LA EDUCACIÓN, UNA INSTANTÁNEA

NACIONAL E INTERNACIONAL

\section{Sistema Inmersivo de realidad virtual basado en cabina y camino sin fin}

Tipo | académico

Descripción | este proyecto busca sentar las bases en la investigación y desarrollo de herramientas para la realidad virtual en el Centro de Innovación y Desarrollo Tecnológico en Cómputo (CIDETEC), como parte de la materia y la línea de investigación de realidad virtual. Hasta el momento, los avances son alentadores y se espera que muy pronto se tenga la cabina armada en su totalidad, para impartir clases apoyándose en ella y desarrollar investigaciones tanto propias como de otros investigadores y de alumnos.

Contribución | proporciona al alumno los principios y habilidades fundamentales para el uso y desarrollo de sistemas de realidad virtual y lo familiariza con elementos básicos de trabajo como son simulación, interfaces hombre-máquina y lenguajes de desarrollo como el lenguaje de modelado de realidad virtual (VRML) y Java3D.

Tecnología | realidad virtual, inmersión lenguaje de modelado de realidad virtual (VRML)

Status | activo, en desarrollo

Web | cidetec.ipn.mx/mtc/progacademico/lgac/rv/Paginas/lgacrv.aspx

País | México

Año | 2007

Auspiciante | Centro de Innovación y Desarrollo Tecnológico en Cómputo (CIDETEC), Instituto Politécnico Nacional (IPN)

\section{1 | The Body RV}

Tipo | privado

Descripción | el Body VR es un instrumento de realidad virtual educativa, que desarrolla contenidos al servicio de la educación, a través de alianzas con universidades en los Estados Unidos y en el extranjero, para crear lecciones sobre diversos aspectos del cuerpo humano. Contribución | apoya la enseñanza de la medicina por medio de experiencias interactivas que permiten explorar diferentes áreas del cuerpo humano. Ayuda al proceso de la enseñanza tradicional de la medicina para médicos, radiólogos y cirujanos, por ejemplo, por medio de simulaciones en realidad virtual que permiten practicar la intervención del cuerpo humano y con ello promueve el desarrollo de habilidades indispensables en el campo de la medicina. 
REALIDAD VIRTUAL Y REALIDAD AUMENTADA

EN LA EDUCACIÓN, UNA INSTANTÁNEA

NACIONAL E INTERNACIONAL

Tecnología | realidad virtual

Status | activo y en desarrollo; planea desarrollar simulaciones virtuales que abarquen integralmente todo el cuerpo humano, para 2020.

Web | thebodyvr.com

País | Estados Unidos / China

Año| 2015

Auspiciante | School of Creative Media at City University of Hong Kong

\section{2 | The Interactive Architecture Lab}

Tipo | académico

Descripción | grupo de investigadores multidisciplinario interesados en el comportamiento e interacción de las cosas, entornos y sus habitantes. Áreas de investigación: entornos de respuesta dinámica, diseño cinético y robótica, interfaces multisensoriales, computadoras portátiles y prótesis, el "Internet de las cosas", rendimiento y coreografía. Una herramienta que permite a profesores, alumnos y familias poder crear itinerarios, escenarios y experiencias basadas en la localización.

Contribución | fomenta el diseño y la realización de proyectos individuales y colaborativos; promueve el diseño para el funcionamiento y la interacción por medio de un programa interdisciplinario que permite considerar objetos, espacio, personas y sistemas como posibles intérpretes, utilizando la tecnología interactiva.

Tecnología | realidad virtual, inmersión

Status | activo

Web | interactivearchitecture.org/

País | Reino Unido

Año | 2016

Auspiciante | The Bartlett School of Architecture 


\section{3 | The World of Comenius}

Tipo | académico

Descripción | software educativo que utiliza el innovador controlador de movimiento Leap Motion montado en la cara de un DK2 para detectar las manos y los dedos del usuario, interpretando los gestos como entrada para controlar el entorno virtual. Utiliza la tecnología para cambiar la forma de aprender y comunicarse. Proyecto aplicado como piloto en el Mendelovo gymnázium (escuela secundaria) de Opava, República Checa.

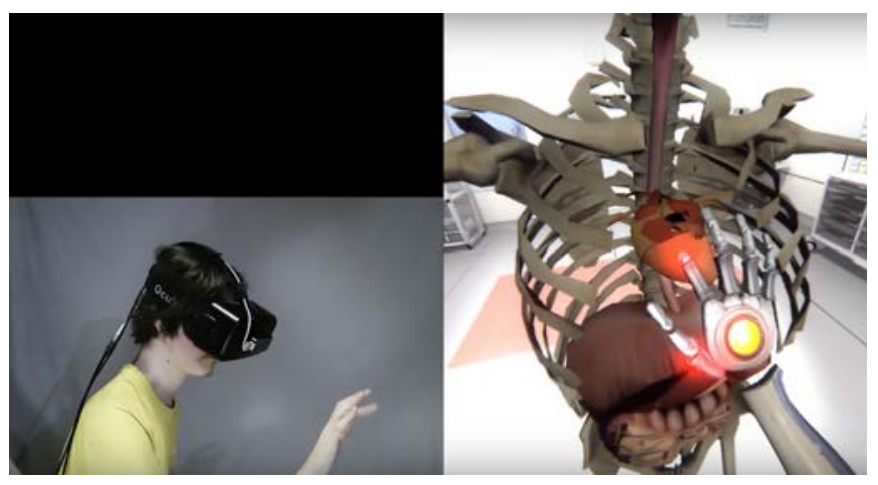

Imagen 15. Conocer y explorar el cuerpo humano en realidad virtual, en The World of Comenius. Foto: Frooxius.

Contribución | acerca una mayor cantidad de contenidos a los estudiantes por medio de una experiencia en la que gracias a la realidad virtual les ofrece cosas que antes no eran posibles, como jugar con átomos y obtener la "sensación" intuitiva de su comportamiento a nivel cuántico; o nadar en una célula o reunirse con personas de la historia y explorar el entorno en el que vivían, al tiempo que tienen la sensación de que están realmente allí.

Tecnología | realidad virtual, inmersión

Status | activo y en desarrollo

Web | frooxius.com/

País | República Checa

Año | 2014

Auspiciante | Tomáš "Frooxius" Mariančík 


\section{4 | UbiCamp}

Tipo | híbrido

Descripción | proyecto que propone una solución integral a los retos de la movilidad virtual para estudiantes y profesores, por medio de acciones como: el desarrollo de un marco que defina criterios de calidad y estándares para la movilidad virtual en la comunidad que integra la organización de Instituciones de Educación Superior (IES), considerando elementos organizativos, tecnológicos, pedagógicos y culturales; creación de recursos educativos y entornos virtuales para el intercambio de experiencias, entre otros.

Contribución | fomenta una rápida y eficaz transmisión de conocimientos, el intercambio de experiencias y la implementación de la movilidad virtual, considerando los recursos tecnológicos, pedagógicos y materiales que se requieren en el proceso de internacionalización de la educación superior.

Tecnología | realidad virtual

Status | proyecto activo y en desarrollo

Web | ubicamp.eu/

País | España

Año | 2014

Auspiciante | Universidad de Oviedo con financiamiento de Erasmus Multilateral Projects

\section{5 | Virtual Cave}

Tipo | híbrido

Descripción | instalación tipo CAVE que tiene la forma de un cubo con dimensiones de 3 x 3 x 3 metros y cuenta con cinco áreas de diseño — las paredes interiores del cubo, incluyendo el suelo. Catorce proyectores que se utilizan para la proyección de los contenidos visuales y el clúster de visualización que consta de ocho equipos. Cuenta con programas para el estudio y la investigación educativos, en áreas como la ciencia forestal, con el programa Sybila (simulador de realidad virtual, de actividad, vida, recursos, impacto ambiental y prospectiva en un bosque). 

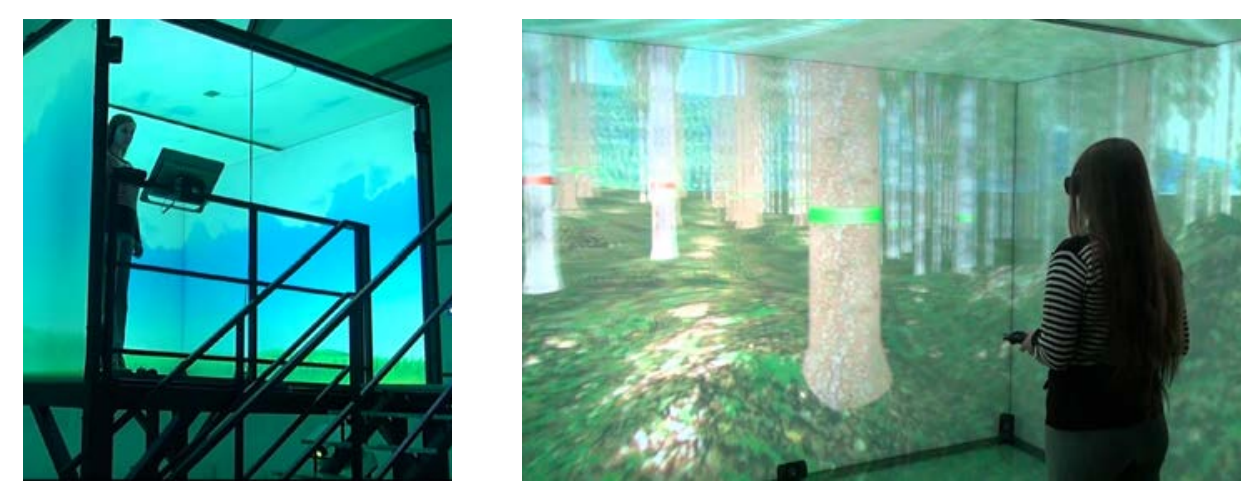

Imagen 16. Cueva virtual en la Universidad Técnica de Zvolen.

Imagen 17. Inmersión en el bosque virtual, Universidad Técnica de Zvolen.

Contribución | permite a los alumnos, profesores e investigadores, examinar los objetos naturales como los bosques y el campo, o los objetos técnicos, como la madera, los propios de la ingeniería, los productos de construcción y toda la tecnología de producción y edificios. Ayuda a mejorar y simplificar el desarrollo y diseño, así como la formación de nuevos métodos y tecnologías para la recolección y procesamiento de datos, y para optimizar la gestión de objetos.

Tecnología | realidad virtual, inmersión

Status | activo

Web | etools.tuzvo.sk/cave/index.html

País | Eslovaquia

Año $\mid 2012$

Auspiciante | Universidad Técnica de Zvolen

\section{6 | Virtual Human Interaction Lab}

Tipo | académico

Descripción | laboratorio de interacciones virtuales que se plantea las siguientes preguntas: ¿Qué nuevos temas surgen a partir del uso equipos de comunicación de realidad virtual immersiva?, ¿cómo puede la realidad virtual ser utilizada para estudiar los matices de las interacciones cara-a-cara?, ¿cómo puede ser utilizada la realidad virtual para mejorar la vida diaria, en conversaciones, empatía y sistemas de comunicación?

Contribución | promueve la investigación sobre la aplicación de la realidad virtual como herramienta de eficacia y mejora del proceso educativo. Permite el acceso al conocimiento, a través de la realidad virtual y las experiencias inmersivas. 
Tecnología | realidad virtual, inmersión

Status | activo

Web | vhil.stanford.edu/

País | Estados Unidos

Año $\mid 2003$

Auspiciante | Stanford University

\section{7 | C6. Virtual Reality Applications Center}

\section{Tipo | académico}

Descripción | instalación educativa en la Universidad Estatal de lowa, que está integrada por seis superficies que en conjunto suman más de 100 millones de píxeles entre todas. La C6 debutó en el año 2000 y recibió una importante actualización en 2008. La C6 original y mejorada fue diseñada e implementada por Mechdyne Corporation.

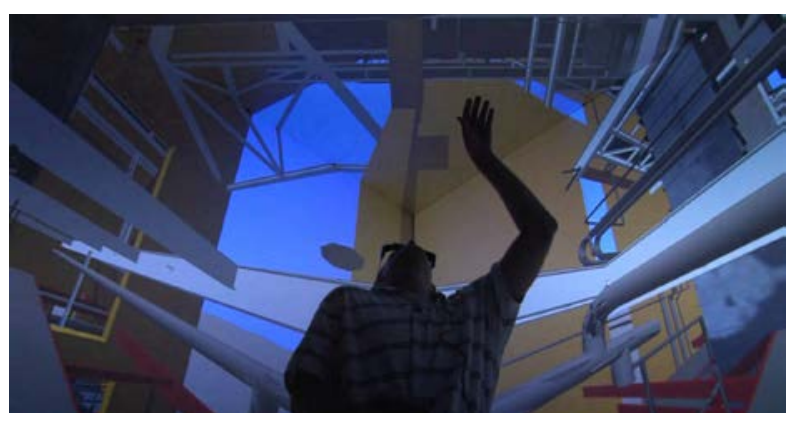

Imagen 18. Experiencia inmersiva en C6.

Contribución | proporciona acceso a un vasto número de contenidos a través de una experiencia inmersiva muy detallada, de alta resolución y con sonido surround de la más alta calidad. De lo más desarrollado en materia de escenarios para inmersión.

Tecnología | Inmersión, realidad virtual

Status | activo

Web | vrac.iastate.edu/

País | Estados Unidos

Año | 2008 (creado en 2000, pero actualizado y mejorado en 2008)

Auspiciante | lowa State University y Mechdyne Corporation 
REALIDAD VIRTUAL Y REALIDAD AUMENTADA

EN LA EDUCACIÓN, UNA INSTANTÁNEA

NACIONAL E INTERNACIONAL

\section{8 | VirtUAM}

Tipo | académico

Descripción | desarrollo de una plataforma educativa aplicable sobre mundos virtuales.

Contribución | permite crear espacios de uso libre para diversos dominios como la educación o la interacción social, crear y desarrollar proyectos tanto de investigación como de carácter educativo/formativo. Aprovecha las nuevas capacidades de interacción hacia una mayor participación del alumno en el proceso de aprendizaje. Fomenta la transformación radical del proceso de enseñanza/aprendizaje, permitiendo un aprendizaje colaborativo y cooperativo entre los estudiantes.

Tecnología | realidad virtual, inmersión, entornos de simulación en tres dimensiones (3D) Status | activo

Web | aida.ii.uam.es/virtuam/

País | España

Año | 2016

Auspiciante | Universidad Autónoma de Madrid

\section{9 | Wonderlab}

Tipo | académico

Descripción | innovación en nuevos campos computacionales para aplicaciones en diseño y procesos de fabricación. Simulación aunada a la ciencia de los materiales.

Contribución | promueve la investigación de las aportaciones de las nuevas tecnologías en la difusión del conocimiento y el aprendizaje del diseño.

Tecnología | realidad virtual

Status | activo

Web | bartlett.ucl.ac.uk/architecture/programmes/postgraduate/labs/march-architectural-design/wonderlab

País | Reino Unido

Año | 2016

Auspiciante | The Barttlet School of Architecture, University College London 
REALIDAD VIRTUAL Y REALIDAD AUMENTADA

EN LA EDUCACIÓN, UNA INSTANTÁNEA

NACIONAL E INTERNACIONAL

\section{0 | zSpace}

Tipo | privado

Descripción | empresa dedicada al desarrollo de plataformas y aplicaciones de realidad virtual, simulación y realidad aumentada, de contenidos académicos en áreas como la anatomía, las artes, matemáticas, ciencia social, ciencias de la tierra y del espacio, entre otras; el laboratorio de aprendizaje de ZSpace consiste en hardware, software y contenidos educativos. Contribución | permite crear experiencias de inmersión e interactivas, con un acercamiento vivencial a los contenidos de corte académico; ayuda a que el aprendizaje sea un proceso más integral y atractivo, hacia una mejor asimilación de conceptos complejos y la eficaz aplicación de la realidad virtual y la realidad aumentada en el proceso educativo. Promueve el desarrollo de proyectos colaborativos y el intercambio del conocimiento a distintos niveles de la educación formal.

Tecnología | realidad virtual, realidad aumentada

Status | activo, en desarrollo de nuevos proyectos

Web | zspace.com/

País | Estados Unidos

Año | 2011

Auspiciante | zSpace 


\section{Conclusiones}

Quince de las treinta iniciativas que conforman la relación son académicas, seis de la industria privada y las ocho restantes consisten en proyectos híbridos, como se aprecia en la gráfica 1.

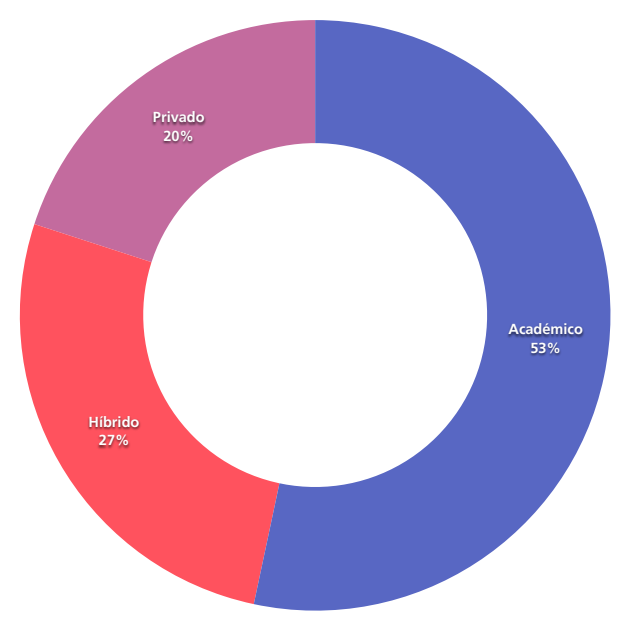

\section{Origen de los proyectos}

En cuanto al lugar de origen de los proyectos de este universo, el $46.6 \%$ proviene de Estados Unidos; $16.6 \%$ de México, $13.3 \%$ de España, 10\% de Reino Unido, 3.3\% de Eslovaquia, 3.3\% de Francia, 3.3\% de Grecia y 3.3\% de República Checa, como se aprecia en la gráfica 2.

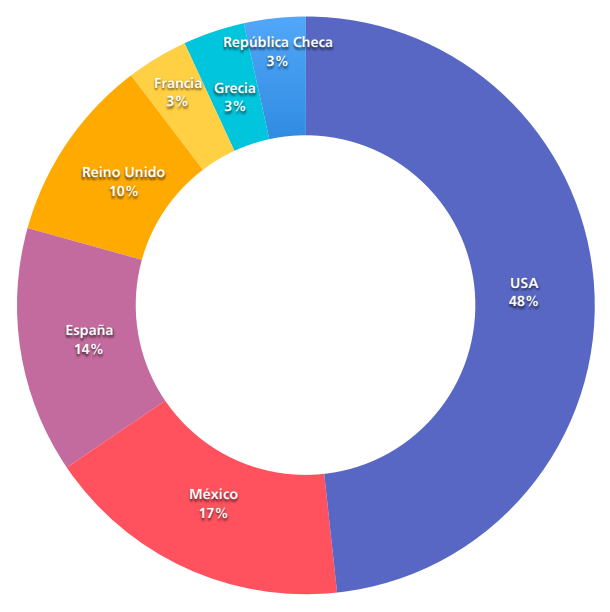


Al revisar el universo de casos, llama la atención el escaso o nulo número de proyectos provenientes de países que destacan por su desarrollo tecnológico e innovación, tales como Alemania, China, Corea del Sur, Finlandia, Francia, Israel, Japón, Rusia, Singapur o Suecia (Browney115, 2017). Estas ausencias son explicables en razón de dos factores clave: por una parte, los sesgos propios de esta investigación, que se concentró en fuentes en inglés y en español; por la otra, el secretismo asociado con las iniciativas de inteligencia militar, sector estrechamente vinculado con el desarrollo de los recursos tecnológicos que nos ocupan. En este orden de ideas, la inclusión de proyectos divulgados en otros idiomas constituye una asignatura pendiente.

Un ejemplo reciente y ampliamente publicitado que no se incluyó en el listado es el antes mencionado Magic Leap, proyecto acerca del cual su fundador, Rony Abovitz, ha mencionado: "el nuestro es un viaje del espacio interior. Estamos construyendo el Internet de presencia y experiencia" (Kelly, 2016). Por el momento se desconoce si tendrá una utilidad específica para el sector educativo o cómo contribuirá al desarrollo de la llamada "enseñanza aumentada" (Dorsfman, 2013), de ahí que no se haya considerado.

Al repasar el listado, observamos que en la mayor parte de los casos el valor agregado del proyecto se relaciona con el acceso a contenidos y experiencias que de otro modo estarían negados para el usuario. Sin embargo, ello nos plantea la interrogante del valor instrumental de estas soluciones: al igual que la visita a una biblioteca análoga no garantiza el involucramiento alumno-docente ni el aprendizaje significativo, la inclusión de realidad virtual o realidad aumentada en la experiencia educativa no garantiza el interés, la motivación o la comprensión del contenido, a menos que estos recursos se utilicen en un entorno educativo adecuado. Decididamente, la información disponible en cada uno de los portales explorados da cuenta de una experiencia de formación distinta (sensorialmente más estimulante, con más detalles, con contenidos inusuales), pero no por ello mejor a la formación fuera de entornos virtuales.

Al respecto, especialistas como Rath (2016), Hong-Xuan (2016), Saltan y Arslan (2016), coinciden en el sentido de que el uso de la realidad aumentada y la realidad virtual sí pueden contribuir a mejorar el proceso educativo. Si ello a su vez implica o no efemeralización deberá verificarse una vez que se haya recuperado, sistematizado y contrastado el análisis de los contenidos, la experiencia de los usuarios (tanto alumnos como docentes) y los resultados del proceso. 
Aunque los sistemas educativos en su conjunto poseen una cantidad llamativa de componentes y aplicaciones que proliferan, no se identificaron elementos suficientes para afirmar que los procesos educativos asociados con los proyectos compilados contribuyeron a la efemeralización educativa, ya que es la conjunción de todos los componentes y actores del ecosistema educativo la que contribuye o no a hacer más con menos y la sola posibilidad de acceder a contenidos que de otra forma serían inaccesibles no nos proporciona suficiente certidumbre a ese respecto. 


\section{Referencias}

Arslan, Ömer \& Saltan, Faith. (2016). The Use

of Augmented Reality in Formal Education: A Scoping

Review. EURASIA Journal of Mathematics Science

and Technology Education, 13(2), 503-520. https://

doi.org/10.12973/eurasia.2017.00628a

Baldwin, J. (1997). BuckyWorks: Buckminster Fuller's Ideas for Today. New York, United States:

JohnWiley \& sons, Inc.

Barnes, C., Johnson, A., Leigh, J., Moher, T., Roussos, M. \& Vasilakis, Ch. (1997). NICE: Combining Constructionism, Narrative, and Collaboration in a Virtual Learning Environment. Recuperado de https:// www.evl.uic.edu/documents/nices97.pdf

Billinghurst, Mark. (2002). Augmented Reality in Education. Recuperado de http://www.solomonalexis. com/downloads/ar_edu.pdf

Browney115 (2017). Most High Tech Countries. The Top Tens. Recuperado de https://www. thetoptens.com/high-tech-countries/

Byrne, Chris M. (1993). Virtual Reality and Education. Recuperado de http://citeseerx.ist.psu.edu/viewdoc/ download?doi=10.1 1.54.6270\&rep=rep1\&type=pdf

Chang, Pan. (2016). Design and Application of Multiscreen VR Technology in the Course of Art Painting. International Journal of Emerging Technologuies in Learning (iJET), 11(9) 56-60. doi http://dx.doi.org/10.3991/ijet.v11i09.6126

Dorsfman, Marcelo (2013). Enseñanza y Tecnologías en el Nivel Superior: La "enseñanza aumentada" y el "docente global". RED Revista de Educación a Distancia, Año XIII, No. 39. Recuperado de http://www.um.es/ead/red/39/dorfsman.pdf

Eggarxou, D. \& Psycharis, S. (2007). Teaching history using a Virtual Reality Modelling Language model of Erechtheum. International Journal of Education and Development using Information and Communication Technology (IJEDICT), Vol. 3, No 3, 115-121. Recuperado de http://ijedict.dec.uwi.edu/ viewarticle. php?id=325\&layout=html

Erner, Guillaume. (2010). Sociología de las tendencias. México: Gustavo Gili.
Fabrika, Marek \& Valent, Peter (2015). Interactive thinning in immersive virtual reality. DVFFA - Sektion Ertragskunde Beiträge zur Jahrestagung, Recuperado de http://sektionertragskunde.fvabw.de/2015/ Beitrag_05.pdf

Hong-Xuan, Bian (2016). Application of Virtual Reality in Music Teaching System. International Journal of Emerging Technologies in Learning is the property of International Association of Online Engineering (IAOE), Vol. 11, No. 11. Recuperado de: http://onlinejournals.org/index.php/i-jet/article/view/6247

Jacobson, Jeffrey. (2008). Ancient Architecture in Virtual Reality; does immersion really aid learning?. Recuperado de http://publicvr.org/publications/ Jacobson2008.pdf

James, P. (2014). 'World of Comenius' Demonstrates Powerful Educational Interaction with Leap Motion and Oculus Rift. Road to VR. Recuperado de http:// www.roadtovr.com/world-of-comenius-educationinteraction-virtual-reality-oculus-rift-leap-motion/

Kelly, Kevin. (2016). The Untold Story of Magic Leap, the World's Most Secretive Startup. Recuperado de https://www.wired.com/2016/04/magic-leap-vr/

Laboratorio Nacional de Visualización Científica Avanzada (LNVCA). (2016). Universidad Nacional Autónoma de México (UNAM) y Universidad Autónoma de Querétaro (UAQ). Recuperado de: http://www.geociencias.unam.mx/ucav/about/lnvca/ Invca.php

Papadopoulos, Ch., Petkov, K., Kaufman, A. \& Mueller, K. (2014). The Reality Deck - Immersive Gigapixel Display. Recuperado de http://www3.cs.stonybrook. edu/ mueller/papers/CGA-RealityDeck.pdf

Rath, David. (2016). Augmented and Virtual Reality: Where Is the Educational Value?. The Journal, Ed Tech Trends. Recuperado de https://thejournal. com/articles/2016/06/15/augmented-and-virtualreality-where-is-the-educational-value.aspx 
Sherman, W. \& Craig, A. (2003). Understanding Virtual Reality Interface, Application, and Design. California, United States: Morgan Kaufmann Publishers.

Ye, You-tian. (2016). Design and Implementation of Digital Art Teaching System Based on Interactive Virtual Technology. International Journal of Emerging Technologies in Learning is the property of International Association of Online Engineering (IAOE), Vol. 11, No. 11. Recuperado de: https://doi. org/10.3991/ijet.v11i11.6254 